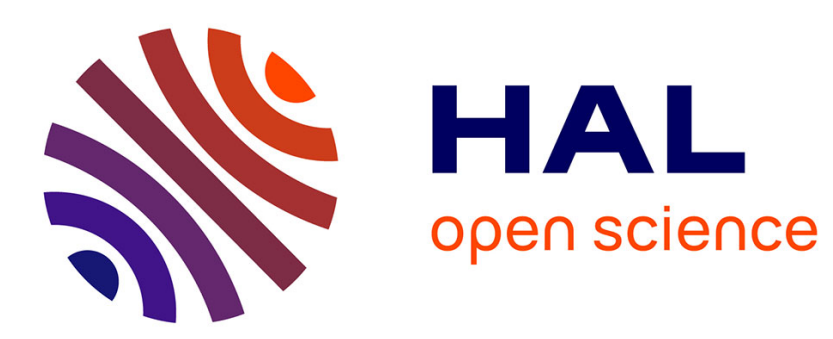

\title{
Real-Time Estimation of Vehicle's Lateral Dynamics at Inclined Road Employing Extended Kalman Filter
}

\author{
Kun Jiang, Alessandro Corrêa Victorino, Ali Charara
}

\section{To cite this version:}

Kun Jiang, Alessandro Corrêa Victorino, Ali Charara. Real-Time Estimation of Vehicle's Lateral Dynamics at Inclined Road Employing Extended Kalman Filter. 11th IEEE conference on Industrial Electronics and Applications (ICIEA 2016), Jun 2016, Hefei, China. pp.2360-2365. hal-01310746

\author{
HAL Id: hal-01310746 \\ https://hal.science/hal-01310746
}

Submitted on 3 May 2016

HAL is a multi-disciplinary open access archive for the deposit and dissemination of scientific research documents, whether they are published or not. The documents may come from teaching and research institutions in France or abroad, or from public or private research centers.
L'archive ouverte pluridisciplinaire HAL, est destinée au dépôt et à la diffusion de documents scientifiques de niveau recherche, publiés ou non, émanant des établissements d'enseignement et de recherche français ou étrangers, des laboratoires publics ou privés. 


\title{
Real-Time Estimation of Vehicle's Lateral Dynamics at Inclined Road Employing Extended Kalman Filter
}

\author{
Kun Jiang, Alessandro Corrêa Victorino, Ali Charara
}

\begin{abstract}
In order to enhance road safety, this article presents a robust methodology for estimation of vehicle's lateral dynamics states, including velocities, sideslip angles, accelerations and tire forces. Awareness of vehicle dynamics states is essential for most intelligent vehicle control systems. However some of these states are not available by direct sensor measurement for technical and economical reasons. Even those available sensors are always coupled with noises or errors. In order to become reliable, many intelligent systems use very expensive sensors, which are not available for ordinary passenger cars. Therefore, it is quite interesting to have an accurate estimation of all dynamics states based on low-cost sensors. The main contribution of this article is providing a reliable estimator of vehicle dynamics states in presence of sensor noises and irregular roads. A best-wheel selection approach is applied in order to have a better understanding of vehicle's driving situation and enable us to select the best models for estimating dynamics states. The proposed estimator is based on the algorithm of extended Kalman Filter, which could minimize the variance of estimation errors. The estimator is implemented in our experimental vehicles and tested at banked track. Experimental results validate and prove the feasibility of this approach.
\end{abstract}

\section{INTRODUCTION}

Accurate knowledge of vehicle dynamics states (such as velocities, yaw rate, sideslip angle and wheel ground forces) is essential for advanced driver assistance systems (ADAS), such as adaptive cruise control (ACC), electric stability control (ESC). The control of longitudinal and lateral dynamics stability can be realized by longitudinal speed control and yaw rate control. The longitudinal speed and yaw rate can be measured directly, thus the control signal could be obtained easily $[2,3]$. However, a system directly controlled by measurement will be probably unstable. The measurement may contain lots of undesired noises and errors. Moreover, the measurement usually cannot cover all the dynamics states of vehicle. In this situation, due to incorrect and inadequate comprehension of vehicle dynamics states, the control system is not able to make the best decisions to ensure vehicle's safety.

Consequently, the objective of this paper is to develop a robust estimator to provide more accurate and more detailed information about vehicle dynamics states than the direct measurement. The dynamics states to be observed include longitudinal speeds, lateral speeds, yaw rate, tire forces and the sideslip angle of vehicle.

The authors are with Sorbonne universités, Université de Technologie de Compiègne, Laboratoire Heudiasyc, CNRS UMR 7253, funded in part by the Region Picardie, VERVE project, MS2T LABEX and ROBOTEX. kun.jiang@hds.utc.fr, acorreau@hds.utc.fr, acharara@hds.utc.fr
Vehicle longitudinal speed sometimes could be regarded as wheel speed, which is measured by wheel speed sensors. Nevertheless, wheel speeds could become unrelated to the vehicle velocity when driving in slippery conditions. In order to compensate the errors caused by wheel slip, GPS signal is used to correct the data of wheel speed sensor as a fusion of data [4-7,9]. Besides the longitudinal speed, sideslip angle is also an important parameter to describe vehicle dynamics. The higher the sideslip angle is, the more easily drift out happens for a vehicle, especially on a low friction road. As a result, the sideslip angle has been used in many control system to improve stability. Two common techniques to estimate vehicle sideslip angle are to directly integrate inertial sensors and to use a physical vehicle model $[5,6]$.

a) Integration: According to kinematic motion of vehicle, the derivative of sideslip angle is a function of lateral acceleration and vehicle velocities, as shown in Equation (1). The advantage of the direct integration method is based on the independence of tire properties, road friction and other vehicle parameters. Nevertheless, the sensor error will be accumulated in the integration process especially for the lateral acceleration signal, which is easily influenced by some internal and external factors.

$$
\dot{\beta}_{c d g}=\frac{a_{y}}{v_{x}}-\dot{\psi}
$$

where $a_{y}$ is the lateral acceleration, $V_{x}$ is the longitudinal velocity, $\dot{\psi}$ is the yaw rate and $\beta_{c d g}$ is the sideslip angle of vehicle at center of gravity (COG).

b) Physical model: The physical model based approach is to estimate the sideslip angle according to the physical vehicle dynamics models. The role of the vehicle dynamics model is to extract the useful information (such as the sideslip angle) from the measurements. The accuracy of this approach is greatly affected by the quality of the dynamics models. In the application, the commonly-used dynamics model is usually a greatly simplified description of the complex vehicle behaviors. The variation of road condition and other vehicle parameters is not taken into account. As a consequent, the model errors is hardly avoidable. A typical vehicle dynamics model is the bicycle model, illustrated in Figure 1.

In order to circumvent the shortcomings of direct integration method and the model-based method, it is necessary to employ sensor fusion. In this way, the dynamics state of vehicle is estimated by the combination of physical model and integration method. Furthermore, during the sensor fusion process, different vehicle dynamics models and sensors can be 
combined to provide a robust estimation. As published in [3], the longitudinal speed is estimated by combining wheel speed sensors and wheel torque sensors. The sensor fusion process can be generally concluded as a process to attribute different weightings or confidence to the measurement of each sensor. The Kalman filter is the most widely-used algorithm to realize the sensor fusion for estimation of vehicle dynamics states. Extended Kalman filter is implemented in [10] to estimate tire/road forces. Dual Kalman filter is proposed in [11] for the estimation of side slip angle and steering stiffness. Particle filter methodology is employed for the sideslip angle in [8].

Until today, developing an accurate estimators for vehicle dynamics states is still a very challenging research topic. That is due to two aspects: 1) the need of an accurate and also simple dynamics model; 2) the need of a robust and time-efficient data processing technique to eliminate unpredictable sensor errors. The main contribution of this paper is to improve the performance of our estimator in both of the two aspects. For the aspect of vehicle modeling, we employed a non-linear tire model and the double track vehicle model, illustrated in Figure 1. Moreover, we have taken into account the vehicle's pitchroll movements and road inclination (both road bank angle and slope angle). For the other aspect, we proposed a best-wheel selection algorithm and combined with the Extended Kalman filter to facilitate the sensor fusion process. In general, we proposed a robust algorithm for real-time estimation of vehicle dynamics states in presence of sensor errors, road angles and tire slips. To evaluate the performance of our estimators, the proposed algorithm is implemented in our experimental vehicle DYNA, a Peugeot 308 [8], as shown in Figure 4.

This paper is organized as follows. Section 2 presents the construction of our proposed estimator. Section 3 introduces the experimental vehicles. Then, experiment results are described in Section 4. Finally, concluding remarks and future perspectives are given in Section 5.

\section{ROBUST ESTIMATION OF VEHICLE'S LATERAL DYNAMICS STATES}

In the literature, the estimator of dynamics states is developed based on the assumption that the inertial sensors are always available. In our context, the sensor failure may occurs. Therefore, the value of acceleration, yaw velocity and linear speed should be estimated with models rather than obtained directly by the measurement. For the simplicity and efficiency, the vehicle dynamics model is divided into 5 subsystems, represented by the 5 blocks in Figure 2. For each of the subsystem, we have developed a Kalman filter to optimize the estimation. The 5 blocks are the pitch-roll angle estimator, the accelerations estimator, the road angle estimator, the velocity estimator and the sideslip angle estimator. The advantage of using 5 Kalman filters is to make the observer more robust, even in presence of sensor errors. The estimation of pitch-roll angle, road angle and velocities are presented in our previous work [1]. In this article, we focus on the robust estimation of lateral dynamics, especially the side slip angles and lateral tire forces. To clearly present the modification we proposed, we concluded our work as two parts: the best wheel selection algorithm and the EKF based sensor fusion algorithm.
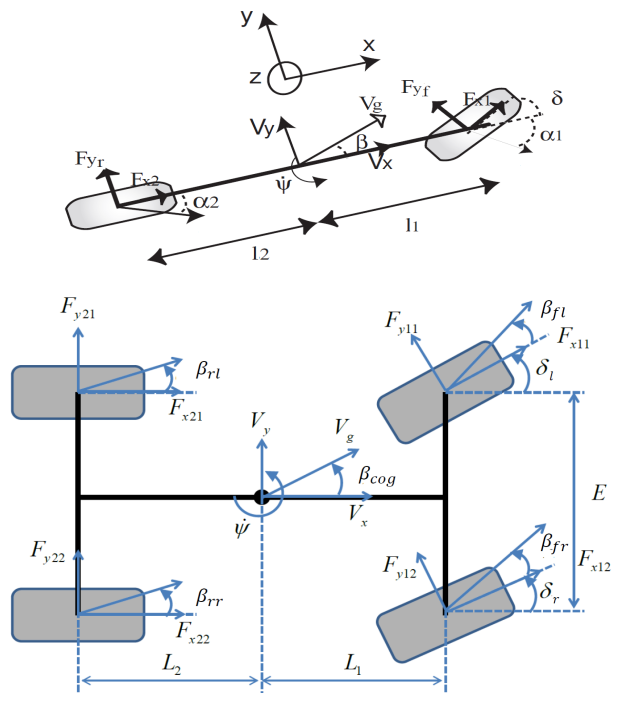

Figure 1. Bicycle Model and Double track model

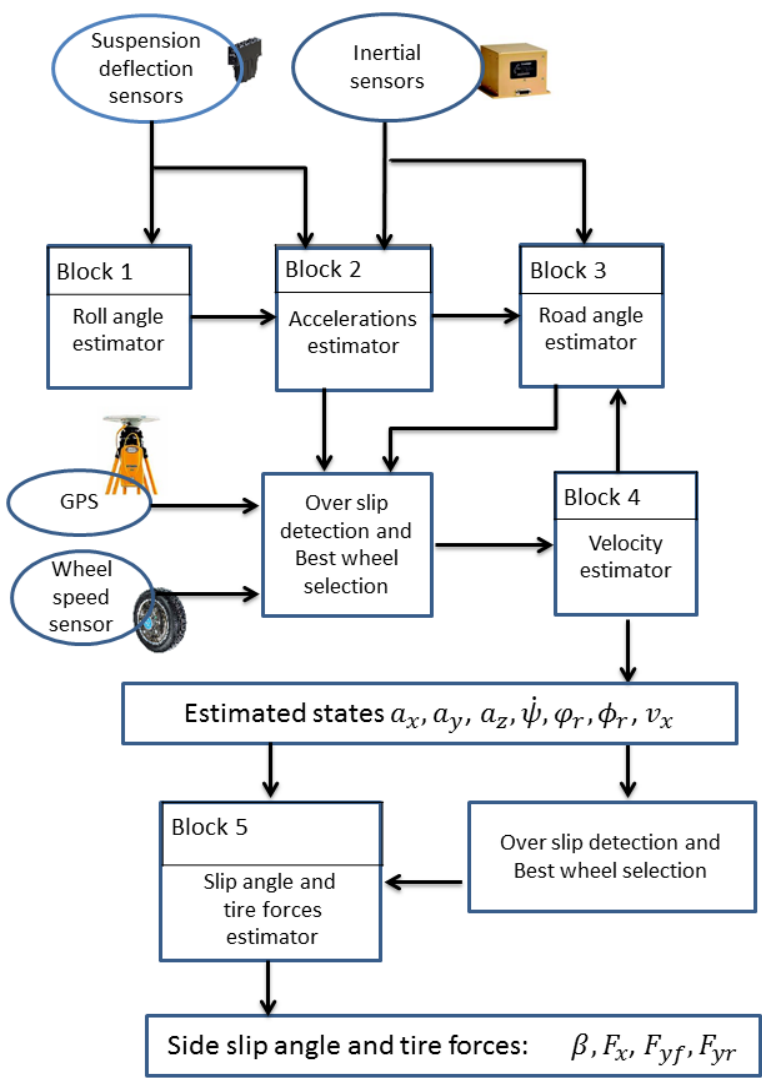

Figure 2. Algorithm overview

\section{A. Best-wheel selection}

To begin with, we remind that the sideslip angle $\beta_{c o g}$ is defined by the Equation (2). Then according to this definition and the double track model, the tire slip angle at each wheel $\left(\alpha_{f l}, \alpha_{f r}, \alpha_{r l}, \alpha_{r r}\right)$ can be calculated by Equation (3).

$$
\beta_{\operatorname{cog}}=\arctan \left(\frac{v_{y}}{v_{x}}\right)
$$




$$
\begin{gathered}
\alpha_{f l}=-\delta_{l}+\beta_{\operatorname{cog}}+\frac{L_{1} \dot{\psi}}{v_{x}} \\
\alpha_{f r}=-\delta_{r}+\beta_{\operatorname{cog}}+\frac{L_{1} \dot{\psi}}{v_{x}} \\
\alpha_{r l}=\beta_{c o g}-\frac{L_{2} \dot{\psi}}{v_{x}} \\
\alpha_{r r}=\beta_{\operatorname{cog}}-\frac{L_{2} \dot{\psi}}{v_{x}}
\end{gathered}
$$

where $v_{x}, v_{y}$ are the lateral and longitudinal speed at COG, $\delta_{l}, \delta_{r}$ are the steering angle of front left and right wheel respectively, the index ${ }_{f l}$ refers to the front left wheel.

Direct measurement of $\beta_{c o g}$ or $v_{y}$ is not available in the ordinary cars. However, the tire slip angle $\left(\alpha_{f l}, \alpha_{f r}, \alpha_{r l}, \alpha_{r r}\right)$ could be estimated with tire models, as introduced in [5] [9]. Thus the problem of estimation of sideslip angle at COG ( $\beta_{\operatorname{cog}}$ ) is transferred into the estimation of tire slip angle at each wheel. Theoretically, all the four tire slip angles can be computed with the tire models, but when the tire slip angle is large, the tire model become very non-linear and less accurate. Therefore, it is better to choose a best-wheel among the four wheels to compute the sideslip angle at COG $\beta_{\text {cog }}$. The best wheel in our context means the wheel with the smallest lateral side slip. The mathematical criterion of selecting the bestwheel is represented by Equation (4)

$$
\beta_{\text {best }}=\left\{\begin{array}{cc}
\delta-\frac{L_{1} \dot{\psi}}{v_{x}}+\alpha_{f} & \text { if }|\delta|-\left|\frac{L \dot{\psi}}{v_{x}}\right|<-\varepsilon \\
\frac{L_{2} \dot{\psi}}{v_{x}}+\alpha_{r} & \text { if }|\delta|-\left|\frac{L \dot{\psi}}{v_{x}}\right|>\varepsilon
\end{array}\right.
$$

where $\beta_{\text {best }}$ is the sideslip angle at COG estimated by the best wheel approach, $\alpha_{f}$ is the slip angles of front tires, $\alpha_{r}$ is the slip angle of rear tires, $\delta$ is the average steering angle, $\varepsilon$ is a constant parameter.

When the front wheels are laterally slipping, the vehicle will be under-steering, $\delta-\frac{L \dot{\psi}}{v_{x}}>0$. As a result, the sideslip angle at COG should be computed with rear wheel slip angles. Otherwise, when the rear wheels are laterally slipping, the vehicle will be over-steering, $\delta-\frac{L \dot{\psi}}{v_{x}}<0$, sideslip angle should be calculated by front wheels. The best wheel approach could eliminate the estimation errors caused by tire slip, due to that the slipping wheel is not used in the estimation. Only the wheel under small slip is chosen for further estimation.

\section{B. Extended Kalman filter based sensor fusion}

Even though the tire slip angle of the best-wheel is small, it is still challenging to accurately calculate its value, as many factors could influence the tire performance, such as temperature or humidity. In the literature, the linear tire model is employed. In order to take into account the non-linearity and the impact of vertical load, we chose the non-linear tire model, Dugoff model, as shown in Equation (5) and illustrated by Figure 3.

$$
\begin{gathered}
F_{y}=C_{\beta} \frac{\beta}{1+\beta} f(\lambda) \\
f(\lambda)= \begin{cases}\lambda(2-\lambda) & ; \lambda \leqslant 1 \\
\lambda & ; \lambda>1\end{cases}
\end{gathered}
$$

where $\lambda$ indicates the tire slip situation, for more details, the reader may refer to [8].

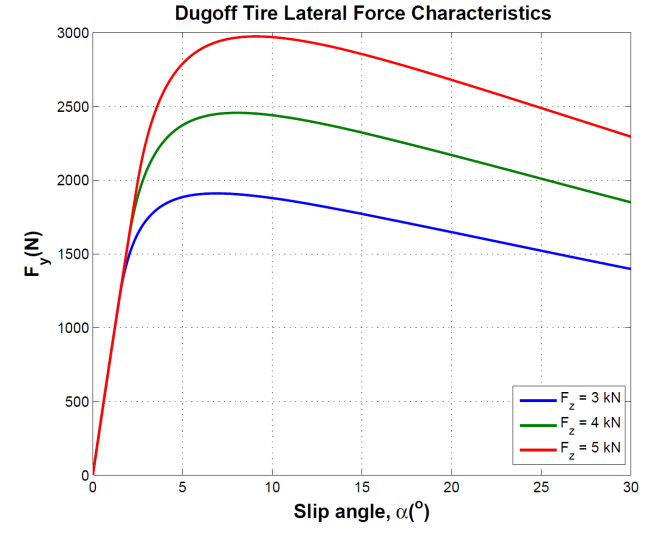

Figure 3. Dugoff lateral force model characteristics

The Figure 3 also explains why we should chose the small slip wheel. When the excessive slip occurs, the tire force can hardly reflect the value of slip angle.

The static tire lateral forces are obtained based on the yaw dynamics and bicycle model, as expressed by Equation (6).

$$
\left.\begin{array}{c}
\ddot{\psi} I_{z} \\
m_{v} a_{y} \\
m_{v} a_{x}
\end{array}\right]=\left[\begin{array}{ccc}
L_{1} \sin \delta & L_{1} \cos \delta & -L_{2} \\
\sin \delta & \cos \delta & 1 \\
\cos \delta & -\sin \delta & 0
\end{array}\right]\left[\begin{array}{c}
F_{x f} \\
F_{y f} \\
F_{y r}
\end{array}\right]
$$

However, the tire lateral forces are not generated instantaneously. Thus the transient tire model is employed to further improve the accuracy of estimation, depicted by Equation (7).

$$
\left[\begin{array}{c}
F_{x f}^{\cdot} \\
F_{y f}^{\cdot} \\
F_{y r}^{*}
\end{array}\right]=\left[\begin{array}{ccc}
0 & 0 & 0 \\
0 & p_{1} & 0 \\
0 & 0 & p_{2}
\end{array}\right]\left[\begin{array}{c}
F_{x f} \\
F_{y f}-\widehat{F}_{y f} \\
F_{y r}-\widehat{F}_{y r}
\end{array}\right]+\operatorname{cov}(\text { noise })
$$

where $\widehat{F} y_{f}$ and $\widehat{F} y_{r}$ are static lateral tire forces calculated by Dugoff models, $p_{1}, p_{2}$ are estimator parameters and configured by experiments.

Substituting the Equation $(5,6,7)$ into (4) generates the sideslip angle at CDG, which is noted as $\beta_{\text {best }}$. The advantage of best-wheel approach is the ability of estimating sideslip angle without integration. However, the estimation is based on simplified tire models, the model errors should not be ignored. Therefore, we employed the EKF algorithm to combine all these estimation methods and models as a sensor fusion. The Kalman filter for estimating sideslip angle can be written as:

$$
\begin{gathered}
{\left[\begin{array}{c}
\dot{\beta}_{c d g} \\
\dot{v}_{y}
\end{array}\right]=\left[\begin{array}{ll}
0 & 0 \\
0 & 0
\end{array}\right] \underset{+\operatorname{cov}(\text { noise })}{\left[\begin{array}{c}
\beta_{c d g} \\
v_{y}
\end{array}\right]+\left[\begin{array}{l}
1 \\
0
\end{array}\right]\left(\frac{a_{y}-g \sin \varphi_{r}}{v_{x}}-\dot{\psi}\right)}} \\
{\left[\begin{array}{c}
\beta_{\text {best }} \\
0
\end{array}\right]=\left[\begin{array}{cc}
1 & 0 \\
v_{x} & -1
\end{array}\right]\left[\begin{array}{c}
\beta_{c d g} \\
v_{y}
\end{array}\right]+\operatorname{cov}(\text { noise })}
\end{gathered}
$$

where $a_{y}, \varphi_{r}, v_{x}, \dot{\psi}, \varphi_{r}$ are estimated in former blocks, and therefore used as constant values in this block. 


\section{EXPERIMENTAL VEHICLE}

\section{A. Vehicle description}

In this section we present the sensors and onboard acquisition system architecture of our experimental vehicle. The experimental vehicle DYNA, a Peugeot 308sw as shown in Figure 4 , is instrumented by the laboratory HEUDIASYC UMR 7253 CNRS at Compiègne, France. This experimental platform is dedicated to validate the algorithm of estimating vehicle dynamics and the embedded real-time systems. All experimental data in this article is acquired by this system. The sensors we used could be generally classified as two categories, sensors used for validation and sensors used for input of the estimator.

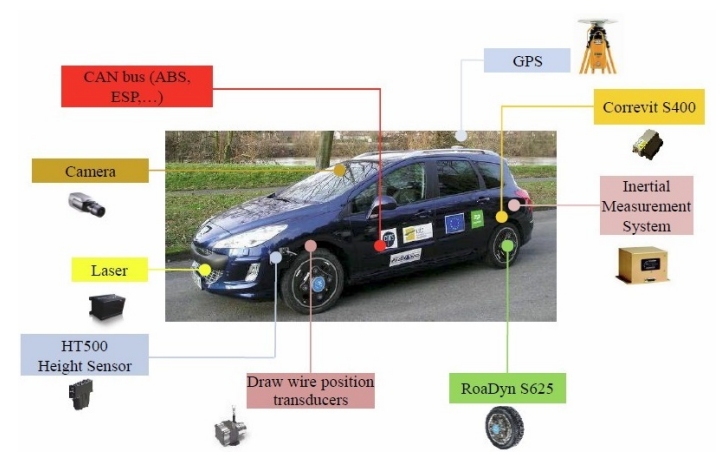

Figure 4. Experimental vehicle DYNA, equipped with different sensors

Sensors used for validation:

CORREVIT S-400: Non-Contact Optical Sensor for measurement of lateral speed and sideslip angle. The sensor is installed at place of the spare wheel under the car.

Kistler RoaDyn S625 wheel force transducers: It's able to measure all the tire-road contact forces and wheel torques in three dimensions. Four wheel force sensors are fixed at each wheel. These sensors are very expensive for ordinary cars. It is noted that they are used only for reference.

A scenario record camera is used to register the vehicle trajectory.

Sensors used for input measures:

CROSSBOWVG700AB: It combines MEMSIC's high performance fiber optic gyros with silicon micro-machined (MEMS) accelerometer technology. It could provide a highly accurate measurement of Vertical Gyro (VG) and Inertial parameters.

CORRSYS-DATRON HT500: it is a non contact distance sensor. It provides measurement of the deflection between chassis and ground. They are installed respectively at four corners of vehicle body.

Available data on CAN bus: wheel rotation velocity, engine speed, yaw rate, brake pressure, lateral acceleration from the ESP, steering wheel angle. And a low-cost GPS.

These devices are located in the trunk of the car with the electric circuit system as shown in Figure 5. The monitoring equipment is located on the back left seat. The operator can manipulate the configuration of the system with a monitor and keyboard.

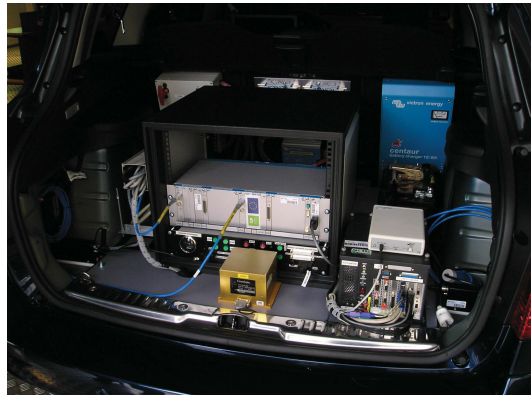

Figure 5. Embedded electronics and computer in the trunk

Sensor data should be sent to a computer which has installed the estimation algorithm. However, some of these sensors cannot be directly connected to the computer. Therefore we developed an acquisition system based on the UEI PowerDNA Ethernet DAQ Cube to fulfill the task. It is capable of acquiring 48 analog channels using a 24-bit converter [8]. It consists of a computer UEI powered by a $200 \mathrm{MHz}$ PowerPC processor running a real-time operating system Xenomai. The Xenomai is a real-time development framework cooperating with the Linux kernel. The software provides data acquisition by using the analog cards AI-217, shown in Figure 6. Digital filters with different cut-off frequency are installed in the cube to cut off high frequency noise. The acquisition frequency of the cube is $4 \mathrm{kHz}$, while the data is sent back in $200 \mathrm{~Hz}$.

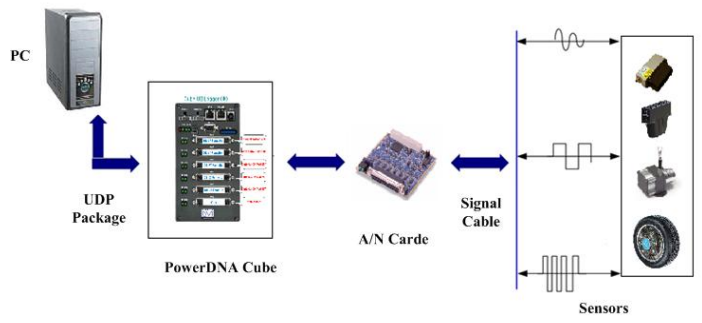

Figure 6. The structure of the acquisition system

\section{B. Software modules}

This car is equipped with an industrial PC in the trunk. A particular prototyping environment, PACPUS ${ }^{1}$, is developed in $\mathrm{C} / \mathrm{C}++$ for the real time estimation system. Following the principle of component-oriented design, PACPUS provides users more versatility in their developments. Different components of PACPUS are developed in our work to fulfill different tasks. For instance, we develop the component "CubeClient" for the task of communication between the cube and the PC. The component is devoted to the task of sending the request, receiving the UDP packet and then decoding the UDP packet. Then the data is sent to the component of vehicle dynamics estimation. Similarly, the components for management of the other sensors are also constructed in our work. The estimation algorithm is developed in the .DLL form as a real-time application. The framework PACPUS employs the Qt API for graphical interfaces and can be integrated with

\footnotetext{
${ }^{1}$ The framework PACPUS is an open source with free license CeCILL-C. It is available at https://devel.hds.utc.fr/software/pacpus/wiki
} 
other development environment for multi-sensor fusion. The schema of PACPUS is shown in Figure 7.

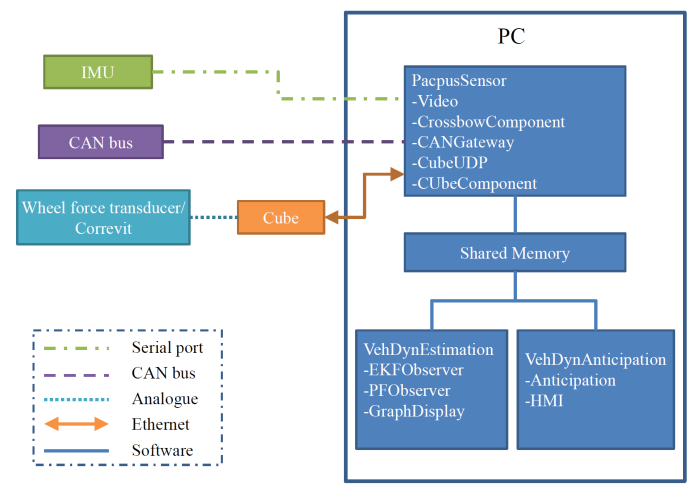

Figure 7. Software architecture of acquisition system and estimation system

\section{EXPERIMENTAL RESULTS}

\section{A. Experimental results of slalom test at flat ground}

The experiments are conducted at a professional vehicle testing ground, UTAC CERAM. Two slalom tests are implemented at flat ground and banked track respectively. During the slalom test at plat ground, the speed is about $60 \mathrm{~km} / \mathrm{h}$, the steering angle range is from -200 to 200 degree, as shown in Figure 8. Estimation of vehicle lateral dynamics at a slalom test is very challenging due to the dramatic variation of vehicle behaviors. Nevertheless, our proposed estimator still provides estimation with satisfactory accuracy and shows good robustness in presence of sensor noises.
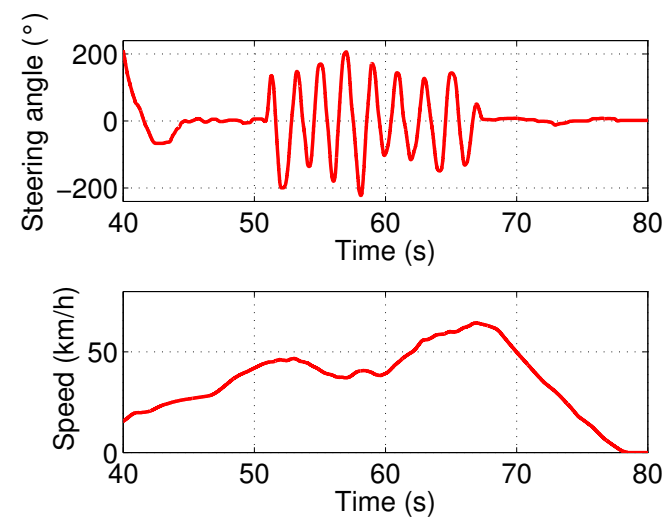

Figure 8. Description of Test 1: slalom test at flat ground

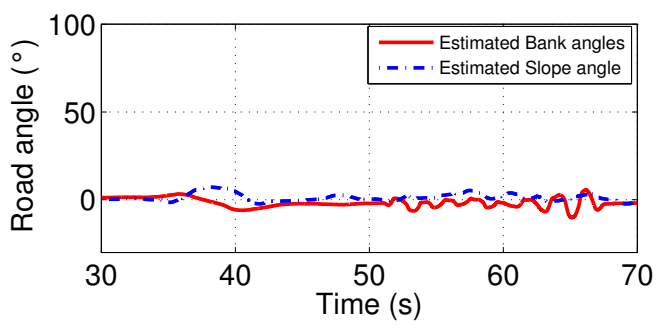

Figure 9. Estimation of road angles. This test is at plat ground, the real value of road angle is close to zero.
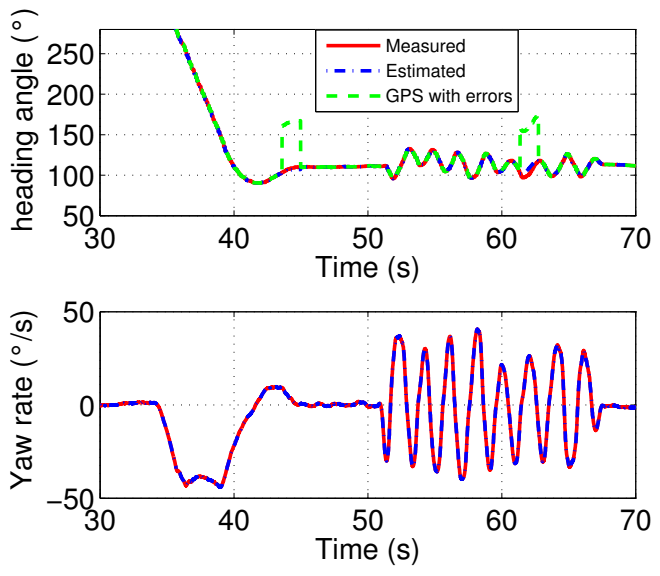

Figure 10. Estimation of heading angle and yaw rate in presence of GPS errors
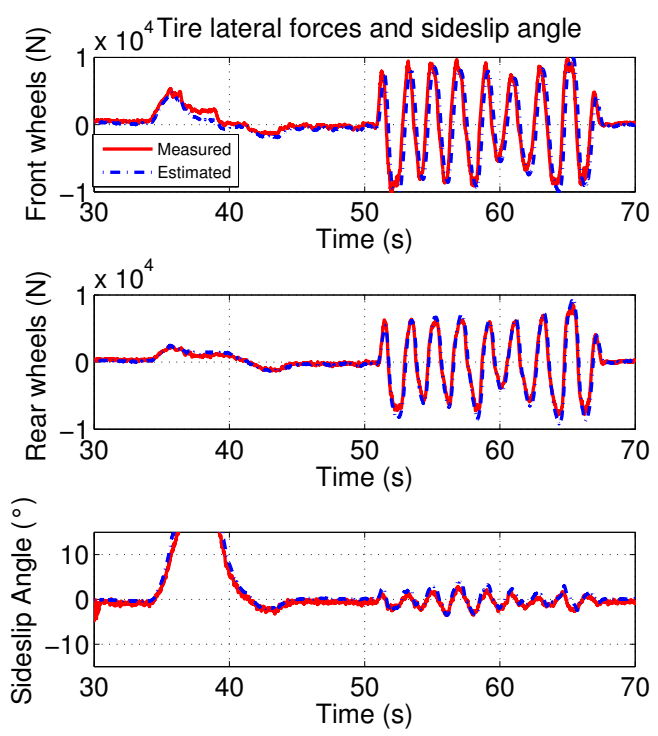

Figure 11. Estimation of lateral dynamics at flat ground: lateral forces of front wheels, lateral forces of rear wheels and side slip angle

Figure 9 shows the result of estimated road angles. The slalom behavior introduced disturbance into the road angle estimation. By using only inertial sensors, the estimation with an error of 5 degree is acceptable. Further improvement can be achieved by fusion with a precise 3D map. Figure 10 shows the estimation of yaw rate and heading angle in presence of GPS errors. The estimated lateral dynamic states are illustrated in Figure 11. The average estimation error is about $3 \%$.

\section{B. Experimental results of slalom test at banked track}

Another test is conducted at banked high speed track. In the testing circuit, there are three parallel tracks: high, middle and low track. According to the information provided by UTAC CERAM, we know the bank angles at high, middle and low track are about 40 degree, 30 degree and 15 degree respectively. In order to hold stable at banked track, the 
vehicle speed reached $135 \mathrm{~km} / \mathrm{h}$, as shown in Figure 12. A slalom behavior is implemented by continuously changing from high track to low track. The estimation results of road angles are shown by Figure 13. When $t=80 \mathrm{~s}-90 \mathrm{~s}$, the vehicle keeps stable at low track, the estimation result is satisfactory. When the vehicle begins to do the slalom behavior, road angle estimation is disturbed but still correctly indicate the variation of road geometry. The lateral tire forces and sideslip angle are illustrated in Figure 14. The average estimation error is about $9 \%$.

\section{CONCLUSIONS AND PROSPECTS}

This paper has presented a new algorithm to estimate vehicle dynamics states, in presence of road angles and sensor errors. To reduce the dynamics model errors, we employed the non linear tire model and modified the double track model by taking into account the road inclination. We also developed an algorithm to detect excessive wheel slip and select the best wheel for estimation of side slip angle. In this way, the estimator stay robust even one wheel is undergoing excessive slip. Furthermore, we employed the extended Kalman filter to combine the estimation results of different approaches, which can be regarded as a process of sensor fusion. Experimental results are presented to evaluate the performance of the new observer.

In the future study, we will focus on improving estimation accuracy with a precise digital map.
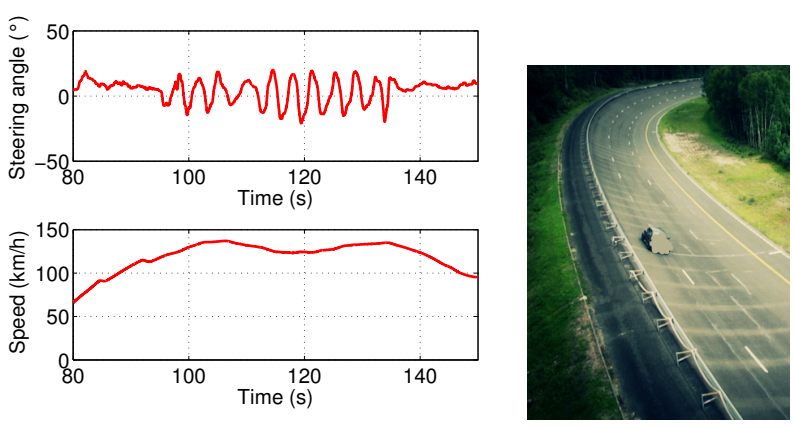

Figure 12. Description of Test 2: slalom test at banked track

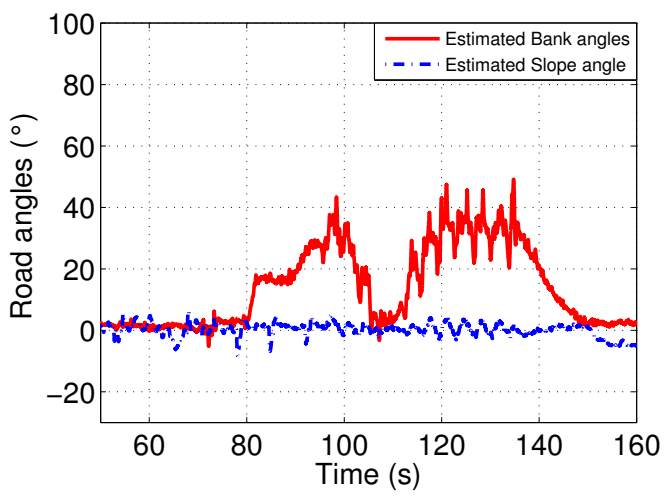

Figure 13. Estimation of road angles. This test is at a banked track, the maximum bank angle is close to $40^{\circ}$.
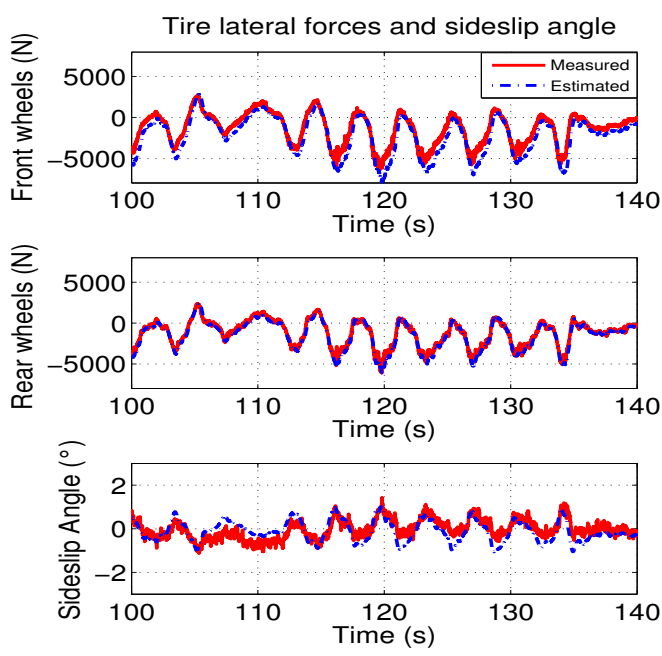

Figure 14. Estimation of lateral dynamics at banked track: lateral forces of front wheels, lateral forces of rear wheels and side slip angle

\section{REFERENCES}

[1] K.JIANG, A.C.VICTORINO, et A.CHARARA. Adaptive estimation of vehicle dynamics through RLS and Kalman filter approaches. Intelligent Transportation Systems (ITSC), 2015 IEEE 18th International Conference on. IEEE, 2015. p. 1741-1746.

[2] Zhu H, Li L, Jin M, Li H, Song J. Real-time yaw rate prediction based on a non-linear model and feedback compensation for vehicle dynamics control. Proc Inst Mech Eng, Part D: J Automob Eng. 2013;227(10):1431- 1445.

[3] M.Klomp, Y.Gao \& F.Bruzelius. "Longitudinal velocity and road slope estimation in hybrid electric vehicles employing early detection of excessive wheel slip", Vehicle System Dynamics: International Journal of Vehicle Mechanics and Mobility, (2014) 52:sup1, 172-188

[4] H.Dahmani, et al. "Vehicle dynamic estimation with road bank angle consideration for rollover detection: theoretical and experimental studies", Vehicle System Dynamics: International Journal of Vehicle Mechanics and Mobility, (2013) 51:12, 1853-1871,

[5] M.Gadola, D.Chindamo, et al. "Development and validation of a Kalman filter-based model for vehicle slip angle estimation " Vehicle System Dynamics 52.1(2014): 68-84.

[6] X.Li, X.Song, C.Chan. "Reliable vehicle sideslip angle fusion estimation using low-cost sensors ."Measurement 51 (2014): 241-258.

[7] M.Ouahi, J.Stéphant and D.Meiezl. "Simultaneous observation of the wheels' torques and the vehicle dynamic state." Vehcicle System Dynamics 51.5 (2013):737-766.

[8] B.Wang, Q. Cheng, A. C.Victorino et al, "Real-Time Experimental Validation of Nonlinear Observer for Vehicle Dynamics Parameters Estimation : A Laboratory Vehicle Description", 2012 IEEE International Conference on Vehicular Electronics and Safety, Turkey, Jul, 2012

[9] R.Jihan, J. Rossetter, and J. Christian. "Vehicle sideslip and roll parameter estimation using GPS." Proceedings of the AVEC International Symposium on Advanced Vehicle Control. 2002.

[10] B. C. Chen et al, "Sideslip angle estimation using extended Kalman filter", Vehicle System Dynamics, vol. 46:S1, pp. 353-364, 2009.

[11] T.A.Wenzel, K.J.Burnham, M.V.Blundell, R.A.Williams. "Dual extended Kalman filter for vehicle state and parameter estimation" Vehicle System Dynamics 44.2(2006)153-171.

[12] Rajamani, R. (2006). Vehicle dynamics and control, Springer.

[13] Ryu, Jihan, and J. Christian Gerdes. "Estimation of vehicle roll and road bank angle." American Control Conference, 2004. Proceedings of the 2004. Vol. 3. IEEE, 2004.

[14] K.Nam, et al. "Estimation of sideslip and roll angles of electric vehicles using lateral tire force sensors through RLS and Kalman filter approaches." Industrial Electronics, IEEE Transactions on 60.3 (2013): 988-1000. 